\title{
SPECTROMETER FOR THE DETERMINATION OF POSITRON AND GAMMA-RAY GASGADE-EMITTING NUCLIDES
}

\author{
K. LJUNGGREN \\ Royal Institute of Technology, Stockholm, 70, Sweden
}

\begin{abstract}
Un spectrometre de coincidence à trois-voies pour la détermination des nuclides qui émettent des positrons ou des cascades de rayons-gamma est décrit. Quelques examples de son utilisation dans les analyses de radioactivation sont donnés.
\end{abstract}

The general procedure in activation analysis work is to separate chemically the elements to be determined. In many cases, however, the results can be obtained more quickly by using the spectral distribution of the radiations emitted. This can be done by spectrometry, either on the irradiated sample itself, in favourable cases, or after a simple chemical separation into groups of elements. The spectrometric or non-destructive activation analysis technique has reached a state of high development with the introduction of multi-channel analysers. It is now possible to make measurements on individual gamma components down to $10^{-11}$ curies, and also on rapidly-decaying nuclides. The interpretation of composite gamma spectra has also been made much easier by various subtraction methods which suppress the Compton distribution or facilitate the consecutive analysis, nuclide for nuclide, of the registered spectra.

Another property of emitted radiations which has not been fully exploited for activation analysis measurements is the time relation between successive quanta or particles emitted by one and the same nucleus. In the great majority of cases the decay of a nucleus involves the emission of more than one particle or photon. The study of these decay properties has led nuclear physicists to the development of an extremely refined measurement technique, based upon the coincidence principle. The principles of coincidence theory and practice are well known, and the different circuits and their data have been dealt with extensively in the literature. Here we need only stress the importance of the scintillation detector in this connection and in nuclear spectrometry. This detector permits millimicrosecond resolving times in the coincidence circuits and has made studies of life-times down to $10^{-11} \mathrm{sec}$ possible. The theory and techniques of the fast coincidence circuits have been excellently reviewed by Bay ${ }^{1}$.

The importance of fast or medium-speed coincidence circuits for the measurements encountered in spectrometric activation analysis work, i.e., selective measurement of one nuclide in a mixture of nuclides, lies in the increased selectivity which can be achieved if the measurement equipment not only selects the required radiation with respect to energy, but also accepts only such signals as are related in a certain way in time. The latter usually means that the signals from two detectors viewing the active source should be simultaneous within a short time interval-the resolution-time 


\section{K. LJUNGGREN}

constant. As the ratio of genuine coincidences to randomly occurring, unwanted coincidence events is improved with a decreasing time constant, the circuits used should be as fast as possible.

Goincidence techniques can be applied to beta-gamma decay, gammagamma cascades, and positron annihilation. The present report deals with some preliminary results obtained in measurements on ${ }^{60} \mathrm{Co}$, which is characterized by a gamma-ray cascade with energies of $1.17 \mathrm{MeV}$ and $1.33 \mathrm{MeV}$ respectively, and ${ }^{64} \mathrm{Cu}$, which is a positron emitter. For the measurements a coincidence spectrometer, based on the post-coincidence energy selection principle originated by Bell and Petch ${ }^{2}$ was used. The principle of the circuit is shown in Figure 1.

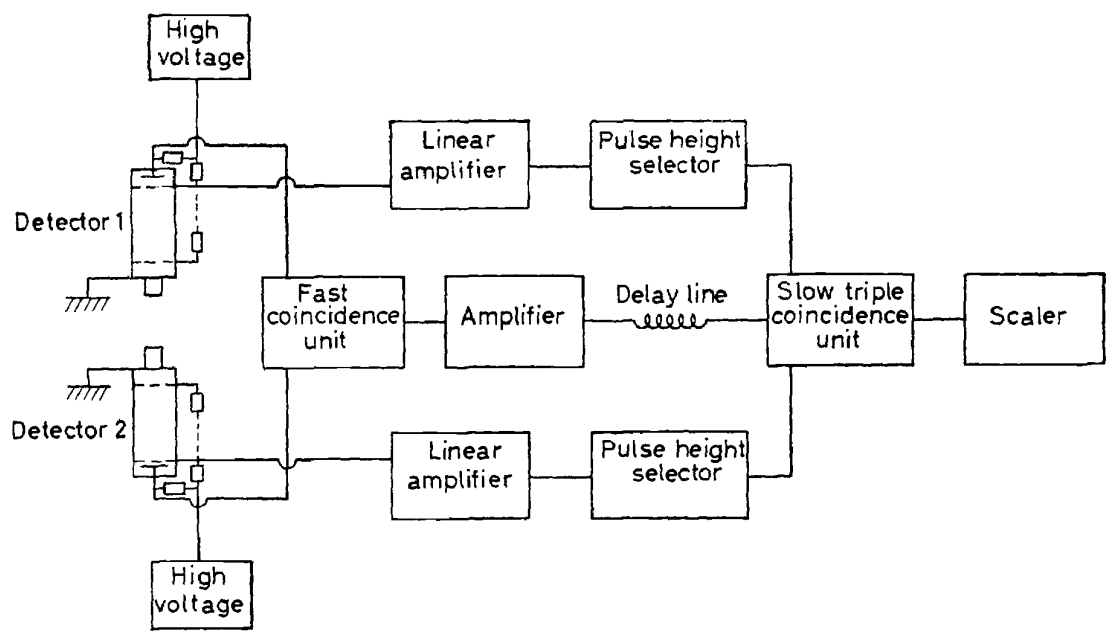

Figure 1. Fast-coincidence spectrometer

The pulses from the two detectors are fed directly to a fast-coincidence stage. After a certain delay, the coincident output pulses are fed to a slower triple-coincidence unit, where they meet pulses which have been analysed in energy in the ordinary way in a differential discriminator after the necessary amplification in a linear amplifier. If the time delay in the fast channel is properly adjusted, coincidences between all three channels will be recorded when the absorbed photons in the two crystals: (1) have the pre-selected energies set in the single-channel analysers, and (2) are simultaneous within the time-interval set in the fast-coincidence unit. Since resolution is independent of what happens in the side channel, the time necessary for pulse-height analysis will not limit the speed of the circuit. If sodium iodide is used as scintillator (it is suitable-as usual-because of its high gamma-absorption efficiency), the resolving time of this type of circuit may still be as short as $10^{-8} \mathrm{sec}$. Many different circuits can be used in the fast-coincidence stage. The present circuit is based on designs given by Azuma and Lewis ${ }^{3}$ and Macq and Vervier ${ }^{4}$. The collector signal from the photomultiplier cuts off a heavily-conducting high-transconductance tube (actually two E180F's in parallel are used to increase the current). 


\section{POSITRON AND GAMMA-RAY GASCADE-EMITTING NUCLIDES}

The anode current is diverted into the coaxial cable (HH 1500) which is connected as anode load. The resulting positive voltage pulse is clipped by a short-circuited cable of suitable length and then applied to one of the control grids of a 6BN6 gated-beam tube, while the other control grid receives pulses from the other photomultiplier through an identical circuit. The $6 \mathrm{BN} 6$ tube gives no anode output unless both control grids are operated simultaneously by positive-going pulses. The 6BN6 tube coincidence circuit, introduced by Fischer and Marshall ${ }^{5}$, is capable of very short resolving times.

The present investigation has been carried out as a comparison between a medium-speed energy selective two-channel system with a resolving time $t=100 \mathrm{~m} \mu \mathrm{sec}$ and a high-speed three-channel system with $t=28 \mathrm{~m} \mu \mathrm{sec}$. The ruclides ${ }^{64} \mathrm{Cu}$ and ${ }^{60} \mathrm{Co}$ have been chosen as examples of positron and gamma cascade emitters because their determination is often of interest in biological and technical problems. They are frequently accompanied by a heavy background of other radiation in irradiated samples. As typical examples, a disturbing activity of ${ }^{24} \mathrm{Na}$ is assumed to accompany the copper activity to provide an illustration of conditions occurring in biological samples, and a disturbing activity of ${ }^{59} \mathrm{Fe}$ (which has metallurgical significance) is assumed to be present together with the cobalt activity. The circuit was therefore set to accept the ${ }^{64} \mathrm{Cu}$ and ${ }^{60} \mathrm{Co}$ radiations under optimal conditions in two different runs. With the settings for the copper annihilation radiation, measurements were also made on ${ }^{24} \mathrm{Na}$ and ${ }^{137} \mathrm{Cs}$. In the same way, a ${ }^{59} \mathrm{Fe}$ source was measured with the circuit set to accept the two photo-peaks at 1.17 and $1.33 \mathrm{MeV}$ in the side channels. The results have been collected in Table 1 .

Table 1

(Source: crystal spacing $=1 \cdot 6 \mathrm{~cm}$ )

\begin{tabular}{|c|c|c|c|c|c|c|c|c|}
\hline & \multicolumn{5}{|c|}{ Sensitivity (counts $\min ^{-1} \mu \mathrm{c}^{-1}$ ) ${ }^{*}$} & \multicolumn{3}{|c|}{ Selectivity ratios } \\
\hline & ${ }^{64} \mathrm{Cu}$ & ${ }^{24} \mathrm{Na}$ & ${ }^{137} \mathrm{Cs}$ & ${ }^{60} \mathrm{Co}$ & ${ }^{59} \mathrm{Fe}$ & ${ }^{64} \mathrm{Cu} /{ }^{24} \mathrm{Na}$ & ${ }^{64} \mathrm{Cu} /{ }^{137} \mathrm{Cs}$ & ${ }^{60} \mathrm{Co} /{ }^{59} \mathrm{Fe}$ \\
\hline $\begin{array}{l}\text { Two channels } \\
t=100 \mathrm{~m} \mu \mathrm{sec}\end{array}$ & 4500 & 28 & $0 \cdot 13$ & 52 & $4 \cdot 8$ & 160 & 35,000 & 11 \\
\hline $\begin{array}{l}\text { Three channels } \\
t=28 \mathrm{~m}_{/ \mathrm{sec}}\end{array}$ & 4000 & 22 & 0.034 & 49 & 0.93 & 180 & 120,000 & 52 \\
\hline
\end{tabular}

*The sensitivities given for ${ }^{60} \mathrm{Fe}$ and ${ }^{137} \mathrm{Cs}$ refer to source strengths of approximately ro $\mu \mathrm{c}$.

Some interesting observations can be made from the data in Table 1. As is to be expected, the sensitivity for copper is considerably higher than for sodium. However, it can be seen that the sensitivity ratio is very little affected when the resolving time is reduced by switching in the fast channel. The selectivity for copper is mainly accounted for by the energy selection in the side channels. The reason why the number of coincidences does not decrease with the resolving time must be that genuine coincidences appear in the decay of ${ }^{24} \mathrm{Na}$. This we know to be the case, since ${ }^{24} \mathrm{Na}$ has two cascaded gammas, of energy $2.76 \mathrm{MeV}$ and $1.38 \mathrm{MeV}$. The part of the Compton distributions that falls in the selected energy channels contains such coincident events. 


\section{K. LJUNGGREN}

On the other hand, ${ }^{137} \mathrm{Cs}$ does not have any coincidences of this type. The sensitivity, already low at the long resolving time, decreases further following the reduction in resolving time.

The absolute efficiency for ${ }^{60} \mathrm{Co}$ is considerably lower than for ${ }^{64} \mathrm{Cu}$. A much lower percentage of the total disintegrations passes by the side channels. The radiation from ${ }^{5} \mathrm{Fe}$ which contains two photo-peaks of nearly the same energy as ${ }^{60} \mathrm{Co}$, but emitted as alternatives and not in cascade, gives a much lower counting rate. This is further reduced with the resolving time.

We may conclude that the short resolving time has a great bearing on the selectivity of the circuit if the measured source does not contain any coincident radiation of an energy higher than the one selected for the measurement of a given nuclide. If the dominating background is a highly energetic gamma cascade, no improvement will be obtained at lower resolving times.

The sensitivity figure for copper-64 which is given in Table 1 corresponds to an overall efficiency of $\left.2 \times 10^{-3}\right)$. The copper efficiency can be increased by using a smaller distance and broader channels, and the resolving time can presumably be reduced to near $10 \mathrm{~m} \mu \mathrm{sec}$ without undue loss of overall sensitivity. Further studies will be made of the optimal circuit parameters in different measuring problems, and of modifications required in the circuit itself to improve its characteristics. One difficulty is already apparent: the photomultiplier gain will vary with the source strength presented to the detectors. This will change the settings of the single-channel pulse-height selectors with the background accompanying the nuclide measured. A different photomultiplier might have to be used, or else a diode-type fast-coincidence circuit might be used instead of the existing one, since the diode-type circuit operates at a lower voltage level. The photomultiplier could thus be run at a lower voltage, where stability problems are less serious. Furthermore, the total activity that can be presented to the counters is limited to around $10 \mu \mathrm{c}$ with the efficiencies specified here.

\section{Addendum}

Recently, a very interesting development in the field of coincidence spectrometers has been published by Hoogenboom ${ }^{6}$. This instrument should be very useful for the measurement of irradiated activation analysis samples.

The iron standard by means of which the sensitivity and selectivity data given above were obtained contains a certain amount of cobalt, as established in later measurements. The cobalt content is about 10 parts/million. With a new ${ }^{59} \mathrm{Fe}$ standard practically free from cobalt new values for the selectivity of the circuit for ${ }^{60} \mathrm{Co}$ relative to ${ }^{59} \mathrm{Fe}$ were obtained: 16 and 140 at resolving times of $100 \mathrm{~m} \mu \mathrm{sec}$ and $28 \mathrm{~m} \mu \mathrm{sec}$ respectively.

\section{References}

1 Z. Bay. I.R.E. Trans. on Nuclear Sci. NS-3, 12 (1956)

2 R. E. Bell and H. E. Petch. Phys. Rev. 76, 1409 (1949)

${ }^{3}$ R. E. Azuma and G. M. Lewis. Phil. Mag. (8) 2, 1325 (1957)

4 P. C. Macq and J. F. Vervier. Rev. Sci. Instr. 28, 843 (1957)

${ }^{5} \mathrm{~J}$. Fischer and J. Marshall. Rev. Sci. Instr. 23, 417 (1952)

${ }^{6}$ A. M. Hoogenboom. Nuclear Instr. 3, 57 (1958) 